\title{
Pathologic complete response after neoadjuvant therapy of breast cancer: pitfalls and shortcomings
}

\author{
Gunter von Minckwitz
}

Published online: 31 March 2012

(C) Springer Science+Business Media, LLC. 2012

Response assessment by imaging methods has always been considered not precise enough and not sufficiently correlated with outcome of patients. In opposite, response assessment by pathological examination was considered to be objective and strongly correlated with a high chance for a cure. Pathologic complete response (pCR) was therefore recommended as the gold standard for response evaluation after neoadjuvant treatment [1]. However, with recent treatment advances, detection of pCR became more frequent and knowledge increased about the potential pitfalls and shortcomings of this surrogate endpoint marker.

Whereas older definitions of pCR referred only to the absence of residual invasive or even gross invasive disease in the breast [2], newer definitions requested also tumorfree axillary nodes or even no residual in situ disease in the breast. This was based on observations in the last decade that patients with no tumor residuals in the breast but involved nodes showed a significant relapse risk [3], and just recently that even patients with in situ breast tumor residuals only showed a higher relapse risk than patients without any residuals in the breast and nodes [4]. Especially the last observation was contradictory to the beliefs that in situ disease is not responsive to systemic treatment at all, and that residual in situ disease cannot be the source of subsequent distant metastasis. However, these beliefs have to be revisited due to evidence reported in this issue of the journal that neoadjuvant chemotherapy given together with trastuzumab can eliminate adjacent ductal carcinoma in situ in about $50 \%$ of cases [5]. As residual in situ disease might simply indicate a higher chance of non-detected

G. von Minckwitz ( $₫)$

German Breast Group, c/o GBG Forschungs GmbH, Martin-Behaim-Straße 12, 63263 Neu-Isenburg, Germany

e-mail: gunter.vonminckwitz@germanbreastgroup.de residual invasive disease which might explain the higher relapse risk of these patients observed in larger cohorts [4]. Nevertheless, harmonization of pCR definitions within clinical trials or at least elaborate reporting of the components of residual disease is warranted. Aiming to identify a cohort of patients with a minimum risk for relapse, the most conservative definition should be used.

The increasing understanding of the biology of distinct intrinsic breast cancer subtypes raises the question if the chance to achieve a pCR after specific treatments is comparable as well as if pCR correlates similarly with survival in all subtypes. With regard to chemotherapy, hormonereceptor (HR) status has so far been shown to be a reproducible predictor for $\mathrm{pCR}$ with higher $\mathrm{pCR}$ rates in HRnegative tumors than in HR-positive tumors [6]. It was suspected that $\mathrm{pCR}$ rates might be higher in HR-positive tumors if endocrine treatment is added. Indeed, a small trial presented in this issue of the journal found a significantly higher pCR rate when letrozole was given in combination with chemotherapy compared to chemotherapy alone [7]. However, this is in contrast to larger trials reported earlier by our group and as well the ECTO group again in this issue of the journal where neither tamoxifen [8] nor exemestane [9] in combination with chemotherapy achieved sufficient pCR rates. However, we have learned that $\mathrm{pCR}$ nicely correlates with survival in HR-negative (triple-negative or HER2-positive) tumors, but much less or not at all in HR-positive tumors [4]. So the observed lower pCR rate in HR-positive tumors does not predict an unfavorable prognosis of these patients. Other surrogate endpoint markers for HR-positive tumors are therefore warranted.

A multitude of markers has been proposed to better predict outcome of neoadjuvant chemotherapy, reports on another 4 of these markers are published in this issue of the journal [10-13]. Therefore, some general prerequisites 
should be described for the development and validation of such new markers: preferably markers should be searched for separately in cohorts with either HR+/HER -, HER2+, or triple-negative tumors; new markers should be immediately validated on independent data sets before their release; and markers should not only be correlated with the occurrence of a pCR but also with survival. Otherwise, we will never understand if this marker will be of clinical importance.

Can we therefore use the neoadjuvant model to test new drugs? Also, could it be used for rapid and early drug approval? Currently, this model can be used only to achieve an early signal of efficacy for new drug combinations if restricted to tumors with triple-negative or HER2-positive disease [14] or to identify potential predictive markers for drugs with new mechanisms of action. Unless a quantitative correlation between increments in pCR and gains in survival in these two most promising subtypes have been confirmed on large data sets, we still have to conduct large-scale adjuvant study to fully assess the effect of systemic treatments.

\section{References}

1. Kaufmann M, von Minckwitz G, Smith R et al (2003) International expert panel on the use of primary (preoperative) systemic treatment of operable breast cancer: review and recommendations. J Clin Oncol 21:2600-2608

2. Fisher B, Brown A, Mamounas E, Wieand S et al (1997) Effect of preoperative chemotherapy on local-regional disease in women with operable breast cancer: findings from National Surgical Adjuvant Breast and Bowel Project B-18. J Clin Oncol 15: 2483-2493

3. Kuerer HM, Newman LA, Smith TM et al (1999) Clinical course of breast cancer patients with complete pathologic primary tumor and axillary lymph node response to doxorubicin-based neoadjuvant chemotherapy. J Clin Oncol 17:460-469

4. von Minckwitz G, Untch M, Blohmer JU, et al (2012) Definition and impact of pathological complete response on prognosis after neoadjuvant chemotherapy in various intrinsic breast cancer subtypes. J Clin Oncol. doi:10.1200/JCO.2011.38.8595
5. von Minckwitz G, Darb-Esfahani, Loibl S, et al (2012) Responsiveness of adjacent ductal carcinoma in situ and changes in HER2 status after neoadjuvant chemotherapy/trastuzumab treatment in early breast cancer-results from the GeparQuattro study (GBG 40). Breast Cancer Res Treat. doi:10.1007/s10549011-1621-0

6. Chang J, Powles TJ, Allred DC et al (1999) Biologic markers as predictors of clinical outcome from systemic therapy for primary operable breast cancer. J Clin Oncol 17:3058-3063

7. Mohammadianpanah M, Ashouri Y, Hoseini S et al (2012) The efficacy and safety of neoadjuvant chemotherapy \pm letrozole in postmenopausal women with locally advanced breast cancer: a randomized phase III clinical trial. Breast Cancer Res Treat. doi: 10.1007/s10549-011-1814-6

8. von Minckwitz G, Costa SD, Raab G et al (2001) Dose-dense doxorubicin, docetaxel, and granulocyte colony-stimulating factor support with or without tamoxifen as preoperative therapy in patients with operable carcinoma of the breast: a randomized, controlled, open phase IIb study. J Clin Oncol 19(15):3506-3515

9. Zambetti M, Mansutti M, Gomez P et al (2012) Pathological complete response rates following different neoadjuvant chemotherapy regimens for operable breast cancer according to ER status, in two parallel, randomized phase II trials with an adaptive study design (ECTO II). Breast Cancer Res Treat. doi:10.1007/ s10549-011-1660-6

10. Glück S, Ross JS, Royce M, et al (2012) TP53 genomics predict higher clinical and pathologic tumor response in operable earlystage breast cancer treated with docetaxel-capecitabine \pm trastuzumab. Breast Cancer Res Treat. doi:10.1007/s10549-0111412-7

11. Ono M, Tsuda H, Shimizu C et al (2012) Tumor-infiltrating lymphocytes are correlated with response to neoadjuvant chemotherapy in triple-negative breast cancer. Breast Cancer Res Treat. doi:10.1007/s10549-011-1554-7

12. Marmé F, Werft W, Walter A et al (2012) CD24 Ala57Val polymorphism predicts pathologic complete response to sequential anthracycline- and taxane-based neoadjuvant chemotherapy for primary breast cancer. Breast Cancer Res Treat. doi:10.1007/ s10549-011-1759-9

13. Collie-Duguid ESR, Sweeney K, Stewart KN et al (2012) SerpinB3, a new prognostic tool in breast cancer patients treated with neoadjuvant chemotherapy. Breast Cancer Res Treat. doi:10.1007/ s10549-011-1625-9

14. Kaklamani VG, Siziopikou K, Scholtens D et al (2012) Pilot neoadjuvant trial in HER2 positive breast cancer with combination of nab-paclitaxel and lapatinib. Breast Cancer Res Treat. doi: 10.1007/s10549-011-1411-8 